\title{
Observation of a Velocity Domain Cooling Instability in a Radiative Shock
}

\author{
M. Hohenberger ${ }^{1},{ }^{*}$ D. R. Symes ${ }^{2}$, J. Lazarus ${ }^{1}$, H. W. Doyle ${ }^{1}$, R. E. Carley ${ }^{1}$, A. S. Moore ${ }^{3}$, \\ E. T. Gumbrell ${ }^{3}$, M. M. Notley ${ }^{2}$, R. J. Clarke ${ }^{2}$, M. Dunne ${ }^{2}$, and R. A. Smith ${ }^{1}$ \\ ${ }^{1}$ Laser Consortium, Blackett Laboratory, Imperial College, London SW7 2BZ. United Kingdom \\ ${ }^{2}$ Central Laser Facility, Rutherford Appleton Laboratory, Oxfordshire OX11 OQX. United Kingdom and \\ ${ }^{3}$ Plasma Physics Division, AWE Aldermaston, Reading RG7 4PR. United Kingdom
}

(Dated: January 13, 2010)

\begin{abstract}
We report on experimental investigations into strong, laser-driven, radiative shocks in noble-gas cluster media. Cylindrical shocks launched with several J exhibit strong radiative effects such as increased deceleration and radiative preheat. Using time-resolved propagation data from single-shot streaked Schlieren measurements we observe temporal modulations on shock position and velocity, which we attribute to the thermal cooling instability, an instability which until now has not been observed experimentally.
\end{abstract}

PACS numbers: Valid PACS appear here

Shocks are a common phenomenon in astrophysics and high-energy-density (HED) environments in general. A shock forms when material expands with supersonic speed into an ambient medium, faster than the surrounding material can adapt to the expansion. If the energy deposition initially launching the shock is limited in time, the shock is followed by a rarefaction which eventually catches up with the shock front and a blast wave is formed, often consisting of a thin shell containing much of the swept-up material [1].

An understanding of shocks and the dynamics of thermal and dynamical instabilities in HED plasmas is vital for numerical models of complex plasma systems. In such environments, radiation can lead to fundamental structural and dynamical changes in the system evolution. A shock becomes radiative if the post-shock conditions lead to an efficient cooling rate through radiative energy losses. The radiation is transmitted through the shock shell and, in an optically thin case, is lost from the system. In contrast, if the upstream material ahead of the shock front is optically thick to parts of the emission spectrum, radiation can be reabsorbed leading to preheat and ionization of the material ahead of the shock front. This modifies the shock propagation dynamics and can lead to growth of instabilities [2, 3].

The temporal expansion of a shock radius is often described as a power-law type function of the form

$$
R(t) \propto\left(E_{0} / \rho\right)^{\alpha / 2} t^{\alpha}
$$

where $E_{0}$ denotes the deposited energy per unit length (in cylindrical geometry) and $\rho$ is the mass density. The parameter $\alpha$ is the deceleration parameter determined by the geometry and the energy dissipation in the system, which for cylindrical, adiabatic blast waves is $\alpha=0.5$ [4]. Dissipative processes such as radiation or ionization necessarily reduce the polytropic index, $\gamma$, of the system,

*Electronic address: M.Hohenberger@Imperial.ac.uk and therefore $\alpha$, to a value below the adiabatic solution and the blast wave decelerates more quickly. In case where radiative losses in the shell are sufficiently large such that the shell cannot support itself any longer, it is pushed by the low-density but high-pressure interior of the shock and collapses to high densities. Specifically the transition to this pressure driven snowplow regime and the associated shell-thinning is thought to make the shock more susceptible to radiation-driven instabilities, one of which we address in detail in this paper.

Radiative shocks can be studied experimentally by utilizing the efficient absorption of high-intensity lasers $\left(>10^{14} \mathrm{Wcm}^{-2}\right)$ by gases of atomic or molecular clusters [5]. Furthermore, using short-pulse lasers temporally decouples the initial energy deposition $(<\mathrm{ps})$ from the subsequent evolution of the plasma system $(\geq n s)$. This allows one to study well characterized shocked plasma systems under repeatable experimental conditions and with access to systems with a varying degree of radiation [6-8]. Provided the experimental geometry and key dimensionless parameters can be matched to astrophysical phenomena, clusters can be used to perform laser-driven shock experiments scalable to astrophysical systems [6,9].

For the experiment presented in this paper, clustering was achieved using a gas jet with an orifice diameter of $500 \mu \mathrm{m}$ and backed with $\mathrm{H}_{2}, \mathrm{Ar}$ and $\mathrm{Kr}$ at 42, 52 and 35 bar, respectively. Weak clustering in $\mathrm{H}_{2}$ made it necessary to operate the gas jet at cryogenic temperatures [10], while clustering in Ar and $\mathrm{Kr}$ was achieved at room temperature. The cluster gas was irradiated using the Vulcan laser at the Rutherford Appleton Laboratory, which provided up to $60 \mathrm{~J}$ on target in a $\lambda=1054 \mathrm{~nm}, 1.4 \mathrm{ps}$ fullwidth-half-maximum (FWHM) pulse focused to $40 \mu \mathrm{m}$ FWHM using an $\mathrm{f} / 18$ off-axis parabola. The focus was aligned into the cluster stream $3 \mathrm{~mm}$ centred above the nozzle. This generated cylindrical shocks over an energy deposition length of $\mathrm{h} \approx 7.5 \mathrm{~mm}$. In a pump-probe geometry, the laser-cluster interaction and subsequent plasma evolution was backlit and imaged using a $\lambda=527 \mathrm{~nm}, 1.3$ ps probe beam providing spatially resolved snapshots of the interaction. To maximise data-output, prior to injec- 
tion into the vacuum chamber the probe beam was divided into three separately timed beams of equal brightness which imaged the interaction onto charged coupled device (CCD) cameras from three different angles orthogonal to the laser propagation direction. Additionally each imaging setup distinguished between orthogonal polarisations, resulting in six (three beamlines $\times$ two polarisations) individually timed probe pulses available on each laser shot. The probe timing could be adjusted from 0 to $15 \mathrm{~ns}$, while the separation between the polarisations was variable from 0 to $3 \mathrm{~ns}$. Each image was then relayed into simultaneous dark-field Schlieren setups and Michelson interferometers [11]. A calorimeter positioned after the laser-cluster interaction region measured absorbed energies while an array of infrared filtered diodes was setup to detect scattered radiation. As in past experiments, no scatter was detected $[5,12]$.

When extracting the shock evolution from time-framed snapshots of the interaction, experimental fluctuations necessarily limit the accuracy to which this can be determined from multi-shot data. As was successfully demonstrated in [13] such ambiguities can be removed by using a long-duration optical backlighter to image the blast wave onto a streak camera via a Schlieren setup, thus tracking the full shock trajectory on a single laser shot. To this end, the nominally 6.8 ns pulse of a q-switched, frequency doubled Nd:YAG laser $(532 \mathrm{~nm})$ was stretched to $\sim 31$ ns FWHM by passing it through a multi-reflection etalon array providing a smooth temporal profile. The elongated pulse was then used to image the plasma onto a Hamamatsu C5680 streak camera via a Schlieren setup. This recorded the shock position with a temporal and spatial resolution of $50 \mathrm{ps}$ and $20 \mu \mathrm{m}$.

Fig. 1 shows typical shock evolution data for $\mathrm{H}_{2}$, Ar and $\mathrm{Kr}$ launched with 5, 6 and $11 \mathrm{~J}$ of deposited energy. Here shock radii expanding away from the nozzle are plotted as a function of time on a double-logarithmic scale. Superimposed onto the data (solid lines) are power-law fits (dashed lines) used to extract time-averaged deceleration parameters. For clarity error bars are only included for $\mathrm{H}_{2}$. As can be seen from Eq. 1, the shock evolution is expected to depend on the deposited energy per unit length divided by the mass density. This should therefore be maintained equal in different gases to allow a direct comparison of the trajectories for identification of potential energy loss mechanisms [14]. In $\mathrm{Ar}$ and $\mathrm{Kr}$, this is the case with $2.3 \pm 0.6 \times 10^{4} \mathrm{Jcm}^{2} / \mathrm{g}$ and $2.9 \pm 0.5 \times 10^{4}$ $\mathrm{Jcm}^{2} / \mathrm{g}$, respectively. However, limitations on the available gas pressure meant that the energy density for $\mathrm{H}_{2}$ exceeded that of the other gases by a factor $\sim 6$. Combined with the knowledge that hydrogen should not lose significant energy through radiation, it is therefore expected to expand faster than the other two cases, as is observed in the data. Interestingly, the extracted deceleration parameter for $\mathrm{H}_{2}$ exceeds 0.5 and therefore the prediction of the adiabatic solution. A contributing factor is the shock front propagating away from the nozzle and therefore down a density gradient. Furthermore,

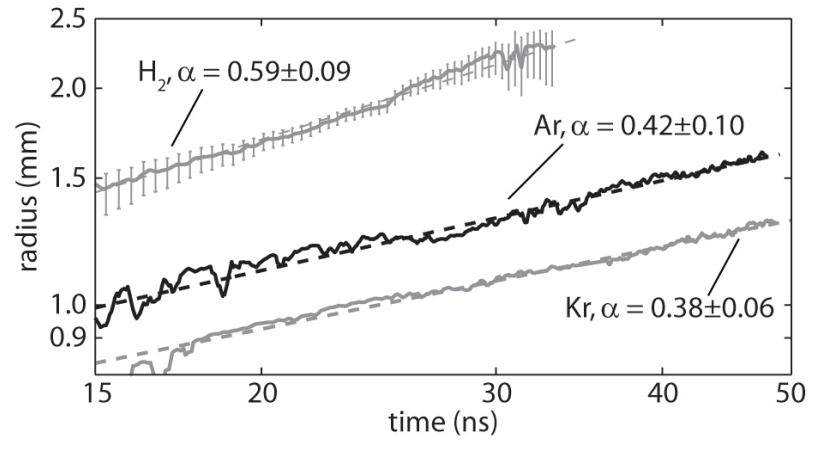

FIG. 1: Streaked Schlieren trajectory results in $\mathrm{H}_{2}$, $\mathrm{Ar}$ and $\mathrm{Kr}$. For clarity, error bars are only included for the $\mathrm{H}_{2}$ data. As expected, $\mathrm{H}_{2}$ expands the fastest, while the deceleration parameter decreases with increasing atomic number.

this could indicate a departure of the shock geometry from a purely cylindrical scenario. In any case, for gases of higher atomic number, the shock deceleration should be increasingly dominated by energy losses through radiation. Indeed, the shock trajectories for $\mathrm{Ar}$ and $\mathrm{Kr}$ for $\mathrm{t}>15$ ns both exhibit an $\alpha<0.5$ suggesting the blast waves are radiative during that time.

This observation is consistent with shocked electron density profiles extracted from short-pulse interferometry data via Abel-inversion. An example for the early time evolution of a shock launched in Ar with $6 \mathrm{~J}$ $\left(1.3 \pm 0.4 \times 10^{4} \mathrm{Jcm}^{2} / \mathrm{g}\right)$ is displayed in Fig. 2. In this plot the shock is propagating from left to right. At $6 \mathrm{~ns}$ (gray line) the shock front, situated at $600 \mu \mathrm{m}$ is already well resolved and the data also exhibits an ionization precursor ahead of the shock front indicative of an energy transport mechanism. At $12 \mathrm{~ns}$ (black line) the shock is significantly more steep and pronounced with the shell thickness $\Delta \mathrm{r}$ having decreased by more than $60 \%$. Additionally, the upstream material ahead of the shock front is heated further, resulting in a doubling of the upstream electron density via increased ionization, while the measured post shock electron density is reduced. From an estimated preheat temperature of a few $\mathrm{eV}$, the electron mean free path is not expected to exceed $20 \mu \mathrm{m}$, such that the extent of the ionization precursor in the data ( $>400 \mu \mathrm{m})$ provides compelling evidence of the strongly radiative nature of these shocks. Using the observed shell thickness, the compression, i.e. the shocked mass density divided by the ambient density, can be estimated to be as high as $C \approx 5.5$ for the late snapshot. This is the highest compression value so far reported in shocks in cluster media and exceeds the theoretical compression limit for an ideal gas $(\mathrm{C}=4)$, further underlining the importance of dissipative processes such as ionization and radiation in shocks under these experimental conditions.

Interestingly, assuming an equal ionization rate the shocked mass in the 12 ns image seems less than would be expected from a simple geometric account of the mass swept up by the shock between the two displayed times. This can, however, be explained by noting that the peak 


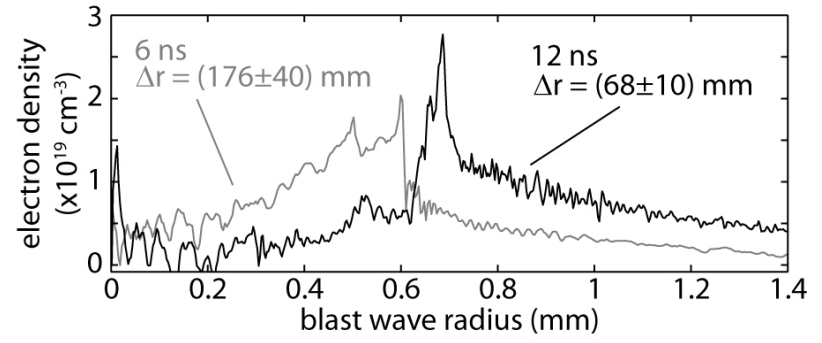

FIG. 2: The early evolution of an Ar shock launched with 6 $\mathrm{J}$ exhibits a strong radiative precursor and significant reduction of the shell thickness $\Delta \mathrm{r}$. Both displayed snapshots were obtained on the same shot.

electron densities generated in the shock front result in very strong and hard to resolve fringe shifts in the interferometric data. Specifically in the data for $12 \mathrm{~ns}$, the fringes are difficult to trace around the peak and the displayed data should be considered a minimum value. It is conceivable that the peak fringe shift is, in fact, underestimated by a factor of $2 \pi$, equivalent to one full fringe shift. This would raise the peak electron density to $\sim 3.5$ $\times 10^{19} \mathrm{~cm}^{-3}$ thus solving the mass discrepancy.

Shell thinning is an important feature in radiative blast waves as it is expected to make the shock more susceptible to instabilities such as the dynamic spatial overstability [3] or the thermal cooling instability (TCI) [2]. While a considerable body of experimental work has been devoted to the former $[7,11,15]$ the latter has received only little experimental attention. In fact, prior to the work presented here it has only been predicted in simulations of astrophysical plasmas and could not be verified experimentally [13]. This instability is thought responsible for a deviation of the ultra-violet and optical emission spectra away from those expected for steady-state flow conditions for example in the Cygnus-loop or the Vela supernova remnant [2]. The TCI occurs when a shock begins to stall while losing kinetic energy through radiation, but then reforms as it expands into the radiatively preheated medium. As a result, this instability is expected to manifest itself in an oscillation of the shock velocity in time, as the shock front periodically gains and loses energy. The onset of the TCI is determined by the radiative cooling function of a gas with a given electron temperature $T_{e}$. This can be described locally following $\Lambda\left(T_{e}\right) \propto T_{e}^{\beta}$ and a shock is expected to be susceptible to the TCI if $\beta$ fulfills the condition $\beta=d(\log \Lambda) / d\left(\log T_{e}\right) \leq 1$ [16]. For astrophysical shocks a shock velocity threshold of $u_{s} \geq$ $120 \mathrm{kms}^{-1}$ has been calculated for the TCI to occur [17]. However, since the instability only depends on the shape of the cooling function with temperature, in principle it should be possible to study this feature on a laboratory scale at a lower temperature and shock velocity provided the condition for $\beta$ is satisfied.

Earlier experimental studies presented in [13] identified $\mathrm{Kr}$ as the most likely candidate to undergo the TCI under the experimental conditions currently accessible in

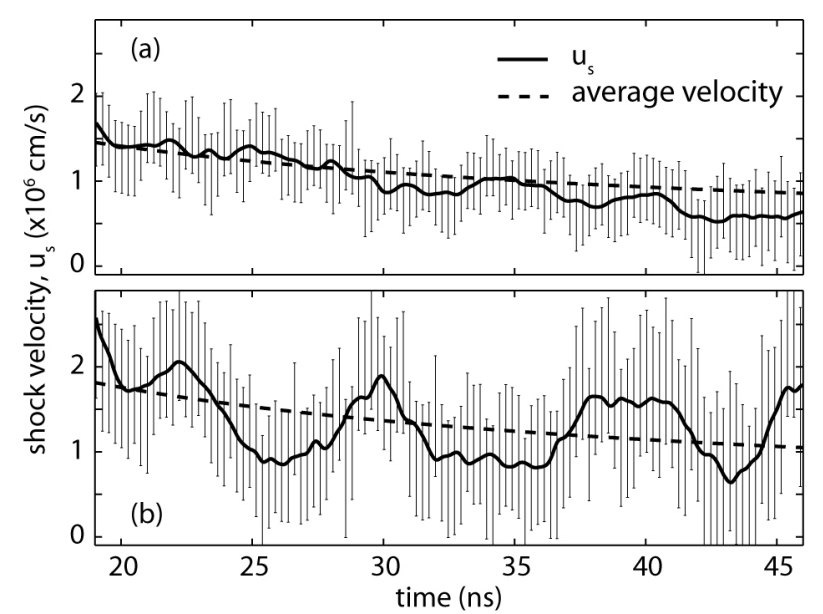

FIG. 3: Temporal evolution of the expansion velocity of cylindrical blast waves in Kr. Both shocks launched with (a) $7 \mathrm{~J}$ and (b) $11 \mathrm{~J}$ decelerate with an averaged $\alpha=0.38$. While (a) shows no unambiguous modulation, (b) exhibits significant oscillations in the shock velocity. This is believed to be caused by the thermal cooling instability.

laser-driven shocks in clusters. However, while at the 1 $\mathrm{J}$ drive level used for previous experiments this could not be demonstrated successfully, it was deemed possible that faster and hotter shocks accessible with higher energy laser systems can potentially enter a regime susceptible to the TCI. A result for the temporal evolution of the shock velocity, comparable to previously obtained data at the $1 \mathrm{~J}$ drive level (see for example [13]), can be seen in Fig. 3(a). It was obtained by averaging the shock propagation from streaked Schlieren measurements in 2 ns intervals, chosen such that the shock front expansion during this time step is well resolved on the streak camera. The Kr shock was launched with a deposited energy of $8 \mathrm{~J}\left(2.1 \pm 0.5 \times 10^{4} \mathrm{Jcm}^{2} / \mathrm{g}\right)$ and for $\mathrm{t}>15 \mathrm{~ns}$, the velocity very closely follows an averaged deceleration with $\alpha=$ 0.38 (dashed line), the deceleration predicted for a fully radiative shock disregarding ionization [18]. While small variations in the shock velocity are visible, these are too small to be interpreted as characteristic oscillations. In stark contrast, Fig. 3(b) shows the shock velocity evolution of a blast wave in Kr launched with slightly more energy $(11 \mathrm{~J})$ and $2.9 \pm 0.5 \times 10^{4} \mathrm{Jcm}^{2} / \mathrm{g}$. While the averaged velocity (dashed line) again corresponds to a deceleration with $\alpha=0.38$, the time resolved velocity now undergoes strong oscillations in time with a period of $\Delta t \sim 7-9 \mathrm{~ns}$.

An estimate of the oscillation time based on the TCI mechanism can be made by calculating the time it takes for the shock to radiate away all of its kinetic energy. Through knowledge of the shock speed, the total kinetic energy contained in the shock in Fig. $3(\mathrm{~b})$ is $\approx 2.4 \mathrm{~J}$. Using the data published in [19], the radiative cooling coefficient at the density of the compressed gas in the shock shell $\left(\sim 10^{19} \mathrm{~cm}^{-3}\right)$ can be extrapolated to be $\sim$ $4.6 \times 10^{-34} \mathrm{Wm}^{3}$, which results in an estimated energy 
loss rate of $\sim 4 \times 10^{8} \mathrm{~J} / \mathrm{s}$ and a cooling time of $\sim 6$ ns. Given that cooling function data is only available for astrophysical densities, this is at most an order-ofmagnitude estimate. Yet, it agrees surprisingly well with the oscillation period of $\Delta t \sim 7-9$ ns visible in the shock velocity in Fig. 3(b).

Following the formalism developed in [18], it is also possible to estimate the energy loss of the shock directly. To this end we use a generalized energy equation which has to be equal on both sides of the shock front, i.e. $E_{1}=E_{2}[20]$ with

$$
E=\rho u\left[\frac{u^{2}}{2}+\frac{\gamma}{\gamma-1} \frac{k_{B} T}{m_{A}}(1+\langle Z\rangle)+E_{e}\right]+\frac{4}{3} u a T^{4}
$$

Here, $\rho, m_{A}, u, \gamma$ and $T$ are the mass density, atomic mass, fluid velocity in the shock frame, polytropic index and temperature, respectively. $\langle Z\rangle$ and $E_{e}$ denote the effective ionization of the gas and the associated excitation energy. The values $k_{B}$ and $a$ are the Boltzmann and radiation constant.

The shock velocity, $u_{1}$, as well as the precursor ionization, $\left\langle Z_{1}\right\rangle$, are extracted directly from the data, while $\rho_{1}$ is obtained from gas jet characterization measurements [21]. Through $\left\langle Z_{1}\right\rangle$, one can estimate $E_{e}$ in the precursor via known ionization potentials [22]. This leaves the preshock polytropic index and temperature, which have to be known in order to calculate the energy content in front of the shock. Assuming local thermal equilibrium (LTE) and following an extrapolation method detailed in [4], an average ionization of $\left\langle Z_{1}\right\rangle=2.9$ gives $T_{1}=4.8 \mathrm{eV}$. Regarding the post-shock values, the gas velocity in the shock frame as well as the post-shock density can be obtained experimentally through measurement of the compression, $C$, and substituting $u_{2}=u_{1} / C$ and $\rho_{2}=C \rho_{1}$. This also gives the effective ionization and, using the same arguments as above, translates to an effective temperature $\left(\left\langle Z_{2}\right\rangle=3.3, T_{2}=6.3 \mathrm{eV}\right)$, leaving only the pre- and post-shock polytropic indices unknown. Naturally, upon choosing the pre-shock value $\gamma_{1}$, the post-shock $\gamma_{2}$ is determined through the requirement for energy balance and Eq. 2. We estimate $\gamma_{1}=1.2[1]$ and find $\gamma_{2}=1.1$.

Ultimately, this allows one to calculate the energy dissipation, $\varepsilon$ due to ionization and radiation via $\varepsilon=$ $\frac{4\left(\gamma_{1}-\gamma_{2}\right)}{\left(\gamma_{1}-1\right)\left(\gamma_{2}+1\right)^{2}}$ and $d E / d t=-\pi R h \rho_{1} u_{1}^{3} \varepsilon$ [18]. This equates to $3.5 \times 10^{8} \mathrm{~J} / \mathrm{s}$, which agrees well with the cooling rate extrapolated from astrophysical values, again confirming an expected oscillation rate on the order of a few ns, as is observed in the experimental data.

The observation of shock velocity oscillations is extremely promising and elegantly demonstrates the capability of the streaked Schlieren technique to observe the TCI. In conjunction with the excellent agreement of the estimated oscillation time with the experimental result, we can say with some confidence that the presented data is, in fact, the first experimental observation of the TCI.

In conclusion, we have presented data from recent experimental investigations of laser-driven shocks in cluster media. The shocks were driven with significantly higher energy densities than in previous experiments and the data clearly shows stronger radiative effects than previously observed in cluster shocks. This includes a reduced deceleration, shell thinning and enhanced compression exceeding the theoretical strong shock limit for an ideal gas. Furthermore, the temporal evolution of shocks in $\mathrm{Kr}$ was investigated by means of the single-shot streaked Schlieren technique. The shock velocity data shows strong oscillations as a function of time, which we believe constitutes the first experimental observation of the thermal cooling instability.

We are pleased to acknowledge useful discussions with R. P. Drake and are grateful for the technical assistance by P. Ruthven, A. Gregory and B. Ratnasekara as well as the Rutherford technical staff and laser operators. This work was supported by EPSRC and MoD UK.
[1] R. P. Drake, High-Energy-Density Physics: Fundamentals, Inertial Fusion, and Experimental Astrophysics, (Springer, New York, 2006)

[2] R. A. Chevalier and J. N. Imamura, Astrophys. J. 261, $543(1982)$

[3] E. T. Vishniac, Astrophys. J. 274, 152 (1983)

[4] Y. B. Zel'Dovich and Y. P. Raizer, Physics of Shock Waves and High-Temperature Hydrodynamic Phenomena (Academic Press, New York, 1966)

[5] T. Ditmire et al., Phys. Rev. Lett. 78, 3121 (1997)

[6] K. Shigemori et al., Astrophys. J. 533, L159 (2000)

[7] M. J. Edwards et al., Phys. Rev. Lett. 87, 085004 (2001)

[8] J. Osterhoff et al., New J. Phys. 11, 023022 (2009)

[9] D. Ryutov et al., Astrophys. J. 518, 821 (1999), D. D. Ryutov et al.. Phys. Plasmas 8, 1804 (2001)

[10] R. A. Smith, T. Ditmire and J. W. G. Tisch, Rev. Sci. Inst. 69, 3798 (1998)
[11] A. D. Edens et al., Phys. Rev. Lett. 95, 244503 (2005)

[12] E. T. Gumbrell et al., New J. Phys. 10, 123011 (2008)

[13] A. S. Moore et al., Phys. Rev. Lett. 100, 055001 (2008)

[14] A. S. Moore et al., Astrophys. Space Sci. 307, 139 (2007)

[15] J. Grun et al., Phys. Rev. Lett. 66, 2738 (1991)

[16] R. S. Sutherland, G. V. Bicknell and M. A. Dopita, Astrophys. J. 591, 238 (2003)

[17] P. A. Kimoto and D. F. Chernoff, Astrophys. J. 485, 274 (1997)

[18] K. A. Keilty et al., Astrophys. J. 538, 645 (2000)

[19] K. B. Fournier et al., Nucl. Fusion 40, 847 (2000)

[20] C. Michaut et al., Eur. Phys. J. D 28, 381 (2004)

[21] T. Ditmire and R. A. Smith, Opt. Lett. 23, 618 (1998)

[22] R. D. Deslattes et al., X-ray Transition Energies Database, National Institute of Standards and Technology (2009) 